\section{Oxidative Stress in Acne Vulgaris}

\section{Amanda Wong ${ }^{1 *}$, Boyang Zhang ${ }^{2}$, Min Jiang ${ }^{3}$, Elaine Gong ${ }^{4}$, Yaochen Zhang ${ }^{5}$ and Sam W Lee}

${ }^{1}$ Beauty Restoration Institute, Jersey City, New York, USA

${ }^{2}$ Department of Environmental Medicine,New York University, New York, USA

${ }^{3}$ Department of Dermatology, Huashan Hospital, Fudan University, Shanghai, China

${ }^{4}$ Ce Lury Health Tech (Shanghai) Co. Ltd., Shanghai, China

${ }^{5}$ Beijing University People's Hospital, Beijing, China

${ }^{6}$ Cutaneous Biology Research Center, Massachusetts General Hospital and Harvard Medical School, Charlestown, MA, USA

\begin{abstract}
Acne vulgaris is one of the most prevalent inflammatory skin conditions found in late childhood and adolescence. Despite various studies, the etiology or pathogenesis of acne is still not well understood and a single, primary cause has not been identified. In the past several decades, various studies have indicated that patients with acne are under increased cutaneous and systemic oxidative stress as indicated by observation of oxidative stress biomarkers. The burden of oxidative stress may not be a mere consequence of acne. Instead, the oxidative stress, lipid peroxidation in particular, may be an early event that helps drive the acne process. Administration of local and systemic antioxidants appears to be effectively against acne. Oxidative stress-initiated inflammation and its maintenance within the pilosebaceous unit are important initial steps for the subsequent pathogenic processes of acne development. The favorable environment created by the oxidation of sebum for Propionibacterium acnes ( $P$ acne) to grow is also an important contributing factor for these steps. This mechanism suggests the oxidative stress as a therapeutic target in the treatment of acne vulgaris.
\end{abstract}

\section{Introduction}

Acne vulgaris (hence forth referred to as acne) is a common dematological disease characterized by hormonally-mediated sebum overproduction, follicular hyperkeratinization and chronic inflammation of the pilosebaceous unit [1]. This disease is frequently found in late childhood and adolescence [2]. Although acne is not a life threatening disease, it causes significant psychological disability

*Corresponding author: Amanda Wong, Beauty Restoration Institute, LLC, Room 1603, 110 River Drive South, Jersey City, New York, USA, Tel: +617 8809626; E-mail: Amanda@BRlinst.com

Citation: Wong A, Zhang B, Jiang M, Gong E, Zhang Y et al. (2016) Oxidative Stress in Acne Vulgaris. J Clin Dermatol Ther 3: 020

Received: March 18, 2016; Accepted: June 09, 2016; Published: June 24, 2016
[3]. In spite of technical advance in experimental dematology, the exact mechanism of the acne process remains elusive. Pilosebaceous units can be numerous on the face, upper back and chest. The sebaceous glands make an oily substance called sebum that normally empties onto the skin surace through the opening of the follicle, which commonly is called a pore. The hair, sebum and keratinocytes that fill the narrow follicle may produce a plug, an early sign of acne. The follicular plugging (comedones) prevents sebum from reaching the skin surface through a pore. The mixture of oil and cells provide a favorable environment for bacteria Propionibacterium acnes ( $P$ acnes) that normally live on the skin to grow in the plugged follicles. These bacteria produce low molecular weight chemotactic factors and enzymes that lead to the accumulation of neutrophils at the site of acne comedones [4]. Because of phagocytosis, these accumulated neutrophils generate Reactive Oxygen Species (ROS). ROS refers to a diverse group of reactive, short-lived, oxygen containing species, such as Superoxide radical $\left(\mathrm{O}_{2}{ }^{*}\right)$, hydrogen peroxide $\left(\mathrm{H}_{2} \mathrm{O}_{2}\right)$, hydroxyl radical $(\cdot \mathrm{OH})$, singlet oxygen $\left({ }^{1} \mathrm{O}_{2}\right)$, , peroxyl radical (LOO'), nitric oxide $\left({ }^{\circ} \mathrm{NO}\right)$ and peroxynitrite $\left(\mathrm{ONOO}^{-}\right)$. The last two sometimes are also referred to as Reactive Nitrogen Species (RNS). These species (ROS and RNS) cause inflammation and tissue injury. Oxidative stress is a signal transducer leading to inflammation. These accumulated neutrophils also release inflammatory factors, such as lysosomal enzymes, which cause inflammation and inflammatory damage to follicular epithelium [5]. Inflammation is a characteristic of tissues to disease or injury and is marked by swelling, redness, heat and pain. When accumulation of tissue injuries breaks down the wall of the plugged follicle, it spills the content to the nearby skin, leading to lesions or pimples. Although the acne pathology involves many factors, a number of studies indicate that oxidative stress caused by imbalance between ROS and antioxidants in favor of ROS is one of the major factors [6-12]. This review summarizes recent development in the role of oxidative stress in the mechanism of acne initiation and its progression.

\section{Oxidative Stress and Skin Tissue Injuries}

Oxidative stress is an imbalance between oxidants (free radicals) and antioxidants in favor of free radicals. Cellular injuries or diseases are caused by the imbalance between production and removal of ROS in the skin. Many cellular processes, including cell metabolism, signaling pathways, inflammation, cell proliferation, and aging, are affected by oxidative stress [13]. Increasing free radicals changes the structure and functions of the proteins, lipids and nucleic acids, and can lead to tissue damages. Products derived from this injury are used as biomarkers of oxidative stress in the assessment and diagnosis of all diseases. ROS in the skin originates from normal cellular metabolism, e.g., mitochondrial respiration and enzymatic activity [13]. Besides, exogenous ROS is generated by various environmental factors, such as UV light or persistent presence of leukocytes, facilitating chronic inflammatory skin conditions. To regulate ROS levels, the skin is rich in enzymatic and non-enzymatic antioxidant defense systems, there by maintaining physiological homeostasis $[14,15]$. 


\section{Free Radicals (Oxidants)}

Because of unpaired electrons, free radicals have a tendency to react with other compounds and act as electron acceptors or oxidizing agents. The main oxidants include ROS and RNS. Internal sources of ROS include mitochondria, peroxisome, inflammatory cells (neutrophils, eosinophils, and macrophages), flavin, adrenaline and dopamine, quinones, the enzyme complex cytochrome P450, $\mathrm{NADPH}$ oxidase and xanthine oxidase. External sources of ROS can be heavy metals, radiation and chemical compounds including anti-cancer drugs, smoking and alcohol. ROS are the most important cellular damage-inducing agents. The first and most important ROS in the body is $\mathrm{O}_{2}{ }^{-}$, which is generated during oxidative phosphorylation reactions and electron transport along with a respiratory chain in the mitochondrial inner membrane (the main source of ROS). The three key members of ROS, $\mathrm{O}_{2}{ }^{*-} \mathrm{H}_{2} \mathrm{O}_{2}$ and $\cdot \mathrm{OH}$ may convert to each other. The $\mathrm{O}_{2}{ }^{*}$ can be converted to $\mathrm{H}_{2} \mathrm{O}_{2}$ through dismutation reaction. $\mathrm{H}_{2} \mathrm{O}_{2}$ can be converted to most reactive ${ }^{\circ} \mathrm{OH}$ radical through metal-mediated Fenton type of reaction $\left(\mathrm{M}^{\mathrm{n}+}+\mathrm{H}_{2} \mathrm{O}_{2} \rightarrow \mathrm{M}^{(\mathrm{n}+1)+}+\mathrm{OH}^{-}+{ }^{\circ} \mathrm{OH}\right)$, where $\mathrm{M}$ represents metal ion in its reduced $\left(\mathrm{M}^{\mathrm{n}+}\right)$ and oxidized form $\left(\mathrm{M}^{(\mathrm{n}+1)+}\right)$. These metals are $\mathrm{Fe}, \mathrm{Cu}, \mathrm{Cr}, \mathrm{V}, \mathrm{Co}$ and Ni.

\section{Antioxidants}

Although the skin possesses an elaborate antioxidant defense system to deal with oxidative stress, excessive and chronic generation of oxidants can overwhelm the cutaneous antioxidant and immune response capacity, leading to oxidative damage resulting in skin injury. A biological antioxidant has been defined as any substance that when present at low concentrations compared to those of an oxidizable substrate significantly delays or prevents oxidation of that substrate [13]. Antioxidants are the body's defense mechanisms against oxidants to maintain balance between oxidants and antioxidants. The most important antioxidant enzymes are Catalase (CAT), Glutathione Peroxidase (GPx) and Superoxide Dismutase (SOD). The most important non-enzymatic antioxidants are vitamin $\mathrm{E}$, vitamin $\mathrm{C}$ (ascorbic acid), vitamin $\mathrm{A}$, flavonoids, albumin, glutathione, thioredoxins, uric acid, metabolites of polyphenols and metal ions chelators such as ferritin and deferoxamine. The main forms of superoxide dismutase in the body include $\mathrm{Zn} / \mathrm{Cu}-\mathrm{SOD}$ and Mn-SOD in the cytoplasm, mitochondria and lysosomes, which catalyze the dismutation of superoxide anion into hydrogen peroxide. Catalase in the peroxisome is the most abundant antioxidant enzyme that causes hydrogen peroxide to degrade into water and oxygen. Glutathione (GSH) is another important antioxidant. GSH together with Glutathione Peroxidase (GPx) comprises a system that maintains the intracellular reducing environment and acts as primary defense against excessive generation of ROS. The oxygen radical scavenging property of GSH directly facilitates ROS neutralization and repairs of ROS-induced damage.

\section{Biomarkers of Oxidative Stress}

The best and most common method to measure free radicals and oxidative stress is to determine the products of the reaction of free radicals with biomolecules as a biomarker. Direct measurement of free radicals and oxidative stress can be achieved by using fluorescent probes to directly capture radicals; however, in an indirect way presence of oxidative stress is measured through the detection of oxidative lesions, as observed on lipids or proteins. The biomarkers of oxidative stress are very important clinically, and the evaluation of body tissue and fluids is used to diagnose pathological conditions, diseases, and cancer types. The Polyunsaturated Fatty Acids (PUFA) in cell membranes are the primary targets of ROS, which are oxidized during the process of Lipid Peroxidation (LPO). The products of LPO, including metabolites such as Malondialdehyde (MDA) and 4-hydroxynoneal (4-HNF), are common indicators of oxidative stress-induced lipid peroxidation or lipid injury. Glutathione is an antioxidant and reducing compound in the body which exists in two forms: resuscitation (GSH) and oxidized (GSSG). In healthy individuals, the highest level of GSH is related to its reduced form (GSH). An increase of GSSG in cells and tissue, therefore, can be a marker of oxidative stress. Studies indicate that GSH decreases in the blood of breast cancer patients, thus acting as a biomarker of oxidative stress. The decreased levels of CAT, GPx and SOD are also indicators of biological system being under oxidative stress.

\section{Oxidative Protein Modification}

The oxidative modification of proteins, lipids and nucleic acids has been implicated in the mechanism of various diseases. Oxidized serum proteins can serve as important in vivo biomarkers of oxidative stress. The in vitro oxidation of amino acid residues results in protein degradation, aggregation and crossing linking. Typically, oxidation of a protein increases the carbonyl contents. This increase is due to the oxidation of Lys, arg, pro or other amino acid residues. Thus, protein carbonyl groups are the biomarkers of oxidative stress [16]. In human plasma, all amino acids in the proteins are susceptible to oxidative modification by oxidants, such as ${ }^{\circ} \mathrm{OH}$ radical. Total serum protein carbonyl contents are significantly increased in moderate to severe acne patients as compared to the total serum protein present in normal human subjects, whereas mild acne patients show negligible increase in the carbonyl contents as compared with healthy individuals [12]. These results show that serum proteins in acne patients are modified by oxidative stress, and this oxidative protein modification may play an important role in acne progression.

\section{Lipid Peroxidation}

Oxidative stress causes damages to all cellular components through attacks on lipids, proteins and DNAs. Among these injuries, lipid damage through oxidative stress-induced lipid peroxidation is particularly relevant to acne [8]. The lipid damage hypothesis in the pathogenesis of acne was proposed half a century ago [17]. In this hypothesis oxidative breakdown of lipid in the skin is not just a consequence of acne process. Lipid peroxides, products of lipid peroxidation, may function as a cause of acne or as an acnegenic agents or both. Based on this hypothesis, antioxidants which decrease oxidative stress and lipid peroxidation would be preventive and therapeutic agents against acne. Topical application of $\alpha$-tocoperol, a lipid soluble antioxidant, reduces the severity of acne [8]. Supporting data for this lipid peroxidation hypothesis comes from a study showing that lipid peroxidation occurs in acne and site specific free radical damage and products of lipid peroxidation might be involved in the initiation of inflammation [18]. Components of sebum, particularly squalene, exhibit enhanced comedogenicity when oxidized [19]. Squalene, which is specific to human sebum, protects skin surface from lipid peroxidation. Squalene is highly sensitive to oxidation. Both squalene and its oxidized products are at much higher levels in acne patients than in healthy individuals [20-23]. Benefit of the synergistic value of oral vitamins $\mathrm{A}$ and $\mathrm{E}$ to decrease lipid peroxidation in acne is observed [24]. Based on this observation, it has been suggested that inflammation in acne is a secondary event following lipid peroxidation [24]. It has also been suggested that 
administration of antioxidants might be a logical and effective means of controlling the inflammation by preventing oxidation of lipids and the subsequent release of lipid peroxidation products into the tissue [24]. This lipid peroxidation hypothesis of acne pathogenesis is supported by several reports of low blood levels of Glutathione Peroxidase (GPx) and its required co-factor selenium to increase lipid peroxidation level among acne patients [25-27]. Various studies indicate that a significant elevation of plasma lipid peroxide levels in acne patients as compared to levels in healthy control populations $[6,8,10,28]$. A recent study qualifies lipid peroxides and inflammatory markers in comedone samples, the results providing further support that lipid peroxidation might be responsible for the progression of acne [29]. Examination of comedo samples indicates that lipid peroxidation is evident in the earliest microcomedo and increases four-fold as inflamed lesions appear [29].

\section{Oxidative Stress}

Oxidative stress biomarkers in the blood are frequently used to study oxidative stress levels in the skin of acne patients. The activities of plasma major antioxidant enzymes, SOD and CAT as well as the level of Total Antioxidant Capacity (TAC) were significantly decreased in acne patients in comparison to those in healthy individuals $[6,10,12]$. It is well established that lipid peroxidation level increases in patients with inflammatory disease [29-31]. MDA, a biomarker of lipid peroxidation is increased in inflammatory cells. Various previous studies shows the high plasma levels of MDA in acne patients $[6,8,10,28]$. Similar to this finding, other studies have shown that serum levels of SOD and CAT in patients with acne were significantly lower than those of healthy individuals $(10,12,32)$. Comparison of SOD levels in patients shows that patients with severe acne have the lowest levels in comparison with patients with mild and moderate acne $[6,12]$. SOD levels are the highest in patients with mild acne [12]. For MDA levels, patients with severe acne shows the highest plasma MDA levels compared with those with mild and moderate acne, suggesting an increase in ROS production overwhelming the antioxidant capacity. The lowest MDA levels were observed in patients with mild acne [12]. GSH levels were lower in samples removed from acne-prone facial areas than uninvolved areas sampled from the medial side of the upper arm of acne patients [33].

Among ROS family members, the level of hydrogen peroxide is used as a marker of oxidative stress. This species are generated by neutrophils via phagocytosis [31]. Similar to other members of ROS, $\mathrm{O}_{2}{ }^{*-}$ and ${ }^{\circ} \mathrm{OH}$, hydrogen peroxide is able to promote inflammation, enhance lipid peroxidation, and cause skin tissue injury [31]. The level of hydrogen peroxide was measured in the blood samples obtained from patients with inflammatory acne and healthy individuals [2]. Patients with inflammatory acne have a $43 \%$ higher level of hydrogen peroxide than normal population. In addition to ROS, reactive nitrogen species are also able to cause oxidative stress and cause skin injury. Nitric oxide (NO) is a free radical and is at the center of these nitrogen species. $\mathrm{NO}$ is involved in the macrophage-dependent killing of intracellular parasites, indicating the potential of this free radical to mediate cytotoxic and pathological effects. The NO level in the tissue is very well regulated. If generated in access, NO can have various toxic effects, depending on its concentration and the microenvironment in which it is generated [34]. There is increasing evidence showing that NO may be involved in the acne pathogenesis $[11,12]$. It has been hypothesized that increased serum level of NO represents another important potential mechanism in the acne pathogenesis [12]. It has been shown that NO level was significantly increased with the increase of acne activity in patient groups with mild, moderate or severe acne [12]. The increased levels of NO observed in the acne patients in the recent study provide evidence of the involvement of nitrosative stress in the progression of acne.

\section{Oxidative Stress, Inflammation and Acne}

Extensive research during last two decades has revealed the mechanism by which continued oxidative stress can lead to chronic inflammation, which in turn could mediate most chronic diseases including cancer, diabetes, cardiovascular, neurological and pulmonary diseases [35]. Oxidative stress can activate a variety of transcription factors including NF- $\kappa B$, AP- $1, \mathrm{p} 53, \mathrm{HIF}-1 \alpha$, PPAR- $\gamma$, $\beta$-catenin/Wnt and Nrf2. Activation of these transcription factors can lead to the expression of over 500 different genes, including those for growth factors, inflammatory cytokines, chemokines, cell cycle regulatory molecules and anti-inflammatory molecules [35]. It has been reported that the release of inflammatory factors is one of the earliest events to occur in the acne process [23]. In addition, oxidative stress within the pilosebaceous unit alters the environment from one that is unsuitable to harbor anaerobic bacteria to one that is perfectly suited for the colonization of such species $[8,23]$. Oxidation of sebum alters oxygen concentration in the follicle, which results in a favorable environment for $P$ acnes to survive. Although further studies are needed, oxidative stress and inflammation set the stage for all subsequent pathogenic factors leading to acne [8].

\section{Heavy Metal and Oxidative Stress}

High concentration of heavy metal contamination in the air is an increasing public health concern in many regions of the world. There is a multitude of anthropogenic emissions in the environment. Generally heavy metals enter into the air due to mining, smelting, fossil fuel combustion (motor vehicles and power plant), municipal waste incineration, cement production and phosphate mining. These metals are in the air as metal-enriched fine Particle Matters (PM). These fine metal containing particles, usually less than 2.5 micrometers in diameter $\left(\mathrm{PM}_{2.5}\right)$ can pose a great health risk for acne patients. Because their small size of approximately $1 / 30^{\text {th }}$ the average width of a human hair, these particle can lodge into the skin. Once these metal ions reach the location of acne sites, such as follicle, these metals will catalyze the reactions of ROS and to convert less reactive species to most reactive ones through the following reactions (reaction [1-3]) [36].

$\mathrm{M}^{\mathrm{n}+}+\mathrm{H}_{2} \mathrm{O}_{2} \rightarrow \mathrm{M}^{(\mathrm{n}+1)+}+\mathrm{OH}^{-}+\cdot \mathrm{OH}$

$\mathrm{M}^{(\mathrm{n}+1)+}+\mathrm{O}_{2}{ }^{-} \rightarrow \mathrm{M}^{\mathrm{n}+}+\mathrm{O}_{2}$

[1] $+[2]$

$\mathrm{H}_{2} \mathrm{O}_{2}+\mathrm{O}_{2} \cdot \stackrel{\mathrm{M}^{\mathrm{n}+} / \mathrm{M}^{(\mathrm{n}+1)}}{\longrightarrow} \mathrm{M}^{(\mathrm{n}+1)+}+\mathrm{OH}^{-}+\cdot \mathrm{OH}+\mathrm{O}_{2}$

In the reactions described above, $\mathrm{M}^{\mathrm{n}+}$ represents transition metal ions in a reduced oxidation state and $\mathrm{M}^{(\mathrm{n}+1)+}$ in an oxidized state. These transition metals are $\mathrm{Fe}, \mathrm{Cu}, \mathrm{Cr}, \mathrm{V}, \mathrm{Co}$ and $\mathrm{Ni}$. These metal catalyzed reactions have two major consequences: (a) Less reactive or toxic $\mathrm{O}_{2}{ }^{-}$ radical can be converted to more reactive and toxic species, $\mathrm{H}_{2} \mathrm{O}_{2}$ and $\cdot \mathrm{OH}$. These species are strong initiator and promotor of lipid peroxidation; (b) Accumulated neutrophils at the site of acne comedones cause phagocytosis, resulting in a large amount of $\mathrm{O}_{2}{ }^{*}$ generated by one electron reduction of molecular oxygen. $\mathrm{O}_{2}{ }^{*}$ can change oxidized metal $\left(\mathrm{M}^{(\mathrm{n}+1)+}\right)$ to reduced metal $\left(\mathrm{M}^{\mathrm{n}+}\right)$. The reduced metal will react with $\mathrm{H}_{2} \mathrm{O}_{2}$ to generate the most reactive ${ }^{\circ} \mathrm{OH}$ radical 
and oxidized metal. In the overall reactions, metal functions as a catalyst. Thus a trace amount of metal ions can generate a large amount of very reactive $\mathrm{H}_{2} \mathrm{O}_{2}$ and $\cdot \mathrm{OH}$. Metal-induced oxidative stress and inflammation are well studied [37]. It appears that metal-contaminated atmosphere enhances oxidative stress, leading to increased lipid peroxidation and increased severity of acne.

\section{Th1 and Th17}

In chronic inflammatory diseases, such as acne the prolonged and persistent production of ROS, which overwhelms cellular antioxidant systems leading to oxidative stress, may influence T-cell function [38]. This contributes to a T-cell phenotype which is hypo-responsive to growth and death signals and persists at the site of inflammation, perpetuating the immune response [38]. Thus, the regulation of T-cell function by oxidative stress therefore has an important implication in acne treatment. Using the samples collected from lesional and non-lesional skin of acne patients a recent study has shown the presence of IL-17A+ cells, which are mainly lymphocytes, in clinically early visible inflamed acne lesions, and also the activation of cytokines, chemokines and antimicrobial peptides known to be typical for the Th17/IL-17 pathway [39]. Th1, Th17 and CD8+ activation and IL-17 related AMP and CXCL chemokine production with neutrophil attraction in acne lesions can be important factors among others in acne pathogenesis [40]. In addition to IL-17A, both Th1 and Th17 effector cytokines, transcription factors and chemokine receptors are upregulated in acne lesions [41]. Il-17A and IL17F are key cytokines for the recruitment and activation of neutrophils and can target keratinocytes, endothelial cells, monocytes and fibroblasts to produce pro-inflammatory mediators such as IL-6, TNF- $\alpha$ and NO [42]. IL-17-related antimicrobial peptide and CXCL chemokine production with neutrophil attraction in acne lesions are thus important factors trigging the inflammation infiltrate [40]. A recent study has shown that airborne PM formed in combustion and thermal processes causes Th17-specific immune responses through persistent free radicals on these particles [43]. Although further studies are needed to link the Th1 and Th17 with oxidative stress, the relationship between oxidative stress and immune response is very established [44].

\section{Causative Role of Oxidative Stress}

It is very well reported that there is an oxidative stress burden in acne patients as shown in various oxidative stress indicators [6-12]. The major question remains to be answered is whether oxidative stress is a causative contributor or a mere consequence of acne. Increasing evidences show that oxidative stress is both a causative factor $[8,18,24,29]$ and that ROS production by neutrophils is involved in the damage of follicular wall to cause lipid peroxidation. The specific lipid that appears to be overproduced in acne is squalene. Higher levels of squalene peroxides, products of squalene peroxidation and reduced vitamin $\mathrm{E}$ are noted in the sebum of acne patients [45]. Squalene peroxides reduce the important skin antioxidant GSH [46], have comedogenic properties, and initiate an inflammation cascade [7]. This cascade begins with exposure of peroxidated squalene to keratinocyte cells, stimulating inflammatory cytokine production and upregulation of lipoxygenase [47]. The synthesis of inflammatory chemoattractant leukotriene B4 increases as lipoxygenase increases, resulting in recruiting neutrophils to generate more ROS. Inhibition of this process will improve acne [48]. The oxidation of sebum alters oxygen tension in the follicle, creating the ideal microaerophilic environment for $P$ acne to grow. $P$ acne does appear to promote inflammation in acne through the production of ROS, mostly $\mathrm{O}_{2}{ }^{-*}$ radicals [49], which can be easily converted to $\mathrm{H}_{2} \mathrm{O}_{2}$ through dismutation reaction and produce $\cdot \mathrm{OH}$ radical if transition metal is present (Equations [1-3]). Various anti-acne drugs and nutrients such as vitamins $\mathrm{E}$ and $\mathrm{C}$, retinol, isotretinoin and zinc sulfate inhibit $P$ acnes-stimulated production of ROS [49]. The effectiveness of these treatments against acne shows that oxidative stress plays an important pathogenesis of acne and in development of therapeutic strategy. It thus appears that oxidative stress-initiated inflammation is the initial step of the subsequent pathogenic processes of acne development.

\section{Antioxidants-based Therapeutic Approach}

Although exact mechanism of oxidative stress in the pathogenesis of acne remains elusive, many researchers believe that it plays a causative role and that clinical intervention with oral or topical agents against oxidative stress are beneficial in acne treatment. At present, there is no large-scale of clinical trial on the therapeutic value of antioxidants against acne. There are various studies in this aspect involved acne patients [8,50]. To achieve the goal of reducing ROS in acne, four approaches can be used: direction scavenging of ROS, enhancement of antioxidant proteins or enzymes, up regulation of antioxidant regulatory proteins and inhibition of ROS generation.

\section{Direct Scavenging of ROS}

Vitamins $\mathrm{A}$ and $\mathrm{C}$ are typical small molecules, which reduce ROS through a direct reaction. Earlier studies have shown that vitamins $A$ and $C$ are beneficial [17]. A recent study used a topical application of green tea against acne [51]. Green tea is a well-studied mixture of natural antioxidants and is widely used one to scavenge ROS. The study shows that applying green tea lotion twice a day reduced total lesion count by $58 \%$ after 6 weeks. A similar study [52] shows that it was more effective than zinc sulfate topical in reduction of acne lesions after weeks. It may be noted that the mechanism of therapeutic action of green tea may not be attributed to its antioxidant function only. Anti-inflammatory ability, anti-microbial activity against $P$ acnes and a potential to reduce sebum production through inhibition of 5 - $\alpha$-reductase may also be important contributing factors.

\section{Enhancement of Antioxidant Proteins or Enzymes}

Zinc ( $\mathrm{Zn}$ ) and Selenium (Se) enhance the activity of certain key antioxidant enzymes, such as SOD and GPx. Only a few studies show the therapeutic value of oral zinc against acne. One of the studies shows that zinc gluconate reduce total inflammatory lesion count by $57 \%$ after two months of treatment [53]. In another study, an oral combination of nicotinamide which support antioxidant pathway, zinc, copper and folic acid taken daily for eight weeks appears to be effective against acne [54]. Lactoferrin, a whey milk protein after removing precipitated casein, has been shown to have an antioxidant effect and have a prominent activity against inflammation in vitro and systemic effects on various inflammatory diseases. The dietary effects of lactoferrin-enriched fermented milk on patients with acne vulgaris, an inflammatory skin condition have been investigated [55]. Patients 18 to 30 years old were randomly assigned to ingest fermented milk with $200 \mathrm{mg}$ of lactoferrin daily ( $\mathrm{n}=18$, lactoferrin group) or fermented milk only ( $\mathrm{n}=18$, placebo group) in a 12-wk, double-blind, placebo-controlled study. Acne lesion counts and grade were assessed at monthly visits. The condition of the skin by hydration, sebum and $\mathrm{pH}$, and skin surface lipids was assessed at baseline and $12 \mathrm{wk}$. The results show that acne showed improvement in the lactoferrin group 
by significant decreases in inflammatory lesion count by $38.6 \%$, total lesion count by $23.1 \%$ and acne grade by $20.3 \%$ compared with the placebo group at $12 \mathrm{wk}$. Furthermore, sebum content in the lactoferrin group was decreased by $31.1 \%$ compared with the placebo group. The amount of total skin surface lipids decreased in both groups. The decreased amount of triacylglycerols in the lactoferrin group was significantly correlated with decreases in serum content, acne lesion counts and acne grade. This study concluded that lactoferrin-enriched fermented milk ameliorates acne vulgaris with a selective decrease of triacylglycerols in skin surface lipids.

\section{Upregulation of Antioxidant Regulatory Proteins}

The Nuclear factor erythroid 2-related factor 2 (Nrf2) pathway plays an imperative role in cellular redox homeostasis and activating this pathway is one of the main defense mechanisms against oxidative or electrophilic stress [56,57]. Nrf2 regulates its target antioxidant proteins through binding of Nrf2 to their Antioxidant Response Element (ARE) in their regulatory regions. These Nrf2 regulatory enzymes are expressed in various isoforms and are distributed in various organelles and subcellular compartments and cooperatively participate in metabolic reactions that eliminate reactive species at their sites of origin. Large-molecule antioxidant and detoxifying enzymes such as Superoxide Dismutase (SOD), glutathione peroxidase, catalase, Glutathione Reductase (GR), Glutamate Cysteine Ligase (GCL), NAD(P) H:Quinone Oxidoreductase1 (NQO1), Heme Oxygenase-1 (HO-1), Glutathione S-Transferase (GST), thioredoxins reductase, peroxiredoxin and sulfotransferase are regulated by Nrf2 and are of prime importance in protecting against oxidative stress at the cellular level. Various natural compounds are inducers of $\mathrm{Nrf} 2$, including oxidizable phenols and quinones, isothiocynates and dithiolethiones [58]. Although activation of Nrf2 by natural compounds can be an excellent approach in reducing the oxidative stress in acne patients, at present this approach has not been investigated in vitro or in vivo.

\section{Inhibition of ROS Generation}

As mentioned above, metal ions are important in generation of $\mathrm{H}_{2} \mathrm{O}_{2}$ from $\mathrm{O}_{2}{ }^{*}$ and ${ }^{\bullet} \mathrm{OH}$ from $\mathrm{O}_{2}{ }^{*}$. Proper chelation of metal ion using chelating agent can block metal ion from catalyzing these reaction. Among various metal chelators, deferoxamine is the most potent to block metal-mediated reactions. This approach is very important in the situation where the air is heavily contaminated by metal ions.

\section{Dietary Approach}

Previous studies have shown that there is a causative role for dietary components in development of acne [40,59]. It has been reported that regional diets with low levels of processed foods and sugar are associated with lower level of acne risk [60]. Clinical studies using low glycemic load meals show beneficial effects [59]. One of the common features for these acne beneficial foods is that they contain a higher amount of plant-derived components or antioxidants. These foods include whole grain, vegetable and fruits.

\section{Conclusions}

The etiology and pathogenesis of acne are not completely understood, and a single, primary cause has not been identified. In the past several decades, various studies have indicated that patients with acne are under increased cutaneous and systemic oxidative stress as indicated by observations of various oxidative stress biomarkers. The burden of oxidative stress may not be simply a consequence of acne. Instead, the oxidative stress, lipid peroxidation in particular, may drive the acne process. Administration of local and systemic antioxidants appears to be effectively against acne. Several studies indicate that oxidative stress-initiated inflammation and its maintenance within the pilosebaceous unit and favorable environment created by the oxidation of sebum for $P$ acne are important initial steps for the subsequent pathogenic processes of acne development. This mechanism suggests the role of oxidative stress as a therapeutic target in the treatment of acne vulgaris.

\section{References}

1. Keyal U, Bhatta AK, Wang XL (2016) Photodynamic therapy for the treatment of different severity of acne: A systematic review. Photodiagnosis Photodyn Ther 14: 191-199.

2. Bergfeld WF (2004) The pathophysiology of acne vulgaris in children and adolescents, Part 1. Cutis 74: 92-97.

3. Gupta MA, Johnson AM, Gupta AK (1988) The development of an acne quality of life scale: reliability, validity, and relation to subjective acne severity in mild to moderate acne vulgaris. Acta Derm Venereol 78: 451-456.

4. Akamatsu H, Horio T, Hattori $\mathrm{K}$ (2003) Increased hydrogen peroxide generation by neutrophils from patients with acne inflammation. Int J Dermatol 42: 366-369.

5. Choi CW, Choi JW, Park KC, Youn SW (2013) Facial sebum affects the development of acne, especially the distribution of inflammatory acne. J Eur Acad Dermatol Venereol 27: 301-306.

6. Al-Shobaili HA (2014) Oxidants and anti-oxidants status in acne vulgaris patients with varying severity. Ann Clin Lab Sci 44: 202-207.

7. Bowe WP, Patel N, Logan AC (2012) Acne vulgaris: the role of oxidative stress and the potential therapeutic value of local and systemic antioxidants. J Drugs Dermatol 11: 742-746.

8. Bowe WP, Logan AC (2010) Clinical implications of lipid peroxidation in acne vulgaris: old wine in new bottles. Lipid Health Dis 9: 141-152.

9. Sahib AS, Al-anbari HH, Abu Raghil AR (2013) Oxidative stress in acne vulgaris: an important therapeutic target. J Mol Pathophysiol 2: 27-31.

10. Arican O, Kurutas EB, Sasmaz S (2005) Oxidative stress in patients with acne vulgaris. Mediators Inflamm 2005: 380-384.

11. Sarici G, Cinar S, Armutcu F, Altinyazar C, Koca R, et al. (2010) Oxidative stress in acne vulgaris. J Eur Acad Dermatol Venereol 24: 763-767.

12. Al-Shobaili HA, Alzolibani AA, AI Robaee AA, Meki AR, Rasheed Z (2013) Biochemical markers of oxidative and nitrosative stress in acne vulgaris: correlation with disease activity. J Clin Lab Anal 27: 45-52.

13. Halliwell B, Gutteridge J (1999) Free Radicals in Biology and Medicine (3rdedn). Oxford University Press, UK.

14. Wagener FA, Carels CE, Lundvig DM (2013) Targeting the redox balance in inflammatory skin conditions. Int J Mol Sci 14: 9126-9167.

15. Godic A, Poljšak B, Adamic M, Dahmane R (2014) The role of antioxidants in skin cancer prevention and treatment. Oxid Med Cell Longev 2014: 860479.

16. Dalle-Donne I, Rossi R, Giustarini D, Milzani A, Colombo R (2003) Protein carbonyl groups as biomarkers of oxidative stress. Clin Chim Acta 329: 23-38.

17. Lorincz Al (1965) Human skin lipids and their relation to skin diseases. Armed Services Technical Information Report AD467008: 1-2.

18. Tapped AL (1974) Lipid peroxidation and fluorescent molecular damage to membranes. In: Trump DF, Arstila A (eds.) Pathological aspects of cell membrane. Academic Press, NY, USA 1: 145-170.

19. Mills OH, Porte M, Kligman AM (1978) Enhancement of comedogenic substances by ultraviolet radiation. $\mathrm{Br} \mathrm{J}$ Dermatol $98:$ 145-150. 
20. Saint-Lager D, Baque A, Cohen E, Chivot M (1986) A possible role for squalene in the pathogenesis of acne. I. In vitro study of squalene oxidation. Br J Dematol 114: 535-542.

21. Hanaoka H, Ohkido A, Hattori Y, Maruta T, Arai T (1971) Reexamination of the sebaceous function with relation to sequalene. Japanese J Dermatol 81: 103.

22. Cotterill JA, Cunliffe WJ, Williamson B, Bulusu L (1972) Further observations on the pathogenesis of acne. Br Med J 3: 444-446.

23. Saint-Leger D, Bague A, Lefebvre E, Cohen E, Chivot M (1986) A possible role for squalene in the pathogenesis of acne. II. In vivo study of squalene oxides in skin surface and intra-comedonal lipids of acne patients. $\mathrm{Br} \mathrm{J}$ Dermatol 114: 543-552.

24. Ayres S Jr, Mihan R (1978) Acne vulgaris and lipid peroxidation: new concepts in pathogenesis and treatment. Int J Dermatol 17: 305-307.

25. Julin L, Edqvist LE, Ekman LG, Ljunghall K, Olsson M (1982) Blood glutathione-peroxidase levels in skin diseases: effect of selenium and vitamin $\mathrm{E}$ treatment. Acta Dermatol Veneraol 62: 211-214

26. Michaëlsson G, Edqvist LE (1984) Erythrocyte glutathione peroxidase activity in acne vulgaris and the effect of selenium and vitamin $E$ treatment. Acta Derm Venereol 64: 9-14.

27. Michaëlsson G (1990) Decreased concentration of selenium in whole blood and plasma in acne vulgaris. Acta Derm Venereol 70: 92.

28. El Garem YF, Ahmed RAM, Ragab MA, Abouzeid AA (2014) Study of oxidative stress in different clinical severities of acne vulgaris. Egyptian J Dermatol Venereol 34: 53-57.

29. Tochio T, Tanaka H, Nakata S, Ikeno H (2009) Accumulation of lipid peroxide in the content of comedones may be involved in the progression of comedogenesis and inflammatory changes in comedones. J Cosmet Dermatol 8 : $152-158$.

30. Akamatsu H, Horio T, Hattori K (2003) Increased hydrogen peroxide generation by neutrophils from patients with acne inflammation. Int $\mathrm{J}$ Dermatol 42 : 366-369.

31. Briganti S, Picardo M (2003) Antioxidant activity, lipid peroxidation and skin diseases. What's new. J Eur Acad Dermatol Venereol 17: 663-669.

32. Kurutas EB, Arican O, Sasmaz S (2005) Superoxide dismutase and myeloperoxidase activities in polymorphonuclear leukocytes in acne vulgaris. Acta Dermatovenerol Alp Pannonica Adriat 14: 39-42.

33. Ikeno $\mathrm{H}$, Tochio $\mathrm{T}$, Tanaka $\mathrm{H}$, Nakata $\mathrm{S}$ (2011) Decrease in glutathione may be involved in pathogenesis of acne vulgaris. J Cosmet Dermatol 10: 240244

34. Brito C, Naviliat M, Tiscornia AC, Vuillier F, Gualco G, et al. (1999) Peroxynitrite inhibits $\mathrm{T}$ lymphocyte activation and proliferation by promoting impair ment of tyrosine phosphorylation and peroxynitrite-driven apoptotic death. $J$ Immunol 162: 3356-3366.

35. Reuter S, Gupta SC, Chaturvedi MM, Aggarwal BB (2010) Oxidative stress inflammation, and cancer: how are they linked? Free Radic Biol Med 49 1603-1616.

36. Lee JC, Son YO, Pratheeshkumar P, Shi X (2012) Oxidative stress and metal carcinogenesis. Free Radic Biol Med 53: 742-757.

37. Leonard SS, Harris GK, Shi X (2004) Metal-induced oxidative stress and signal transduction. Free Radic Biol Med 37: 1921-1942.

38. Bennett SJ, Griffiths HR (2013) Regulation of T-cell functions by oxidative stress. In: Alcaraz MJ, Hualillo O, Sanchez-Pernaute O (eds.). Studies on Arthritis and Joint disorders. Springer Science \& Business Media, Germany. Pg no: 33-48.

39. Kelhälä HL, Palatsi R, Fyhrquist N, Lehtimäki S, Väyrynen JP, et al. (2014) IL-17/Th17 pathway is activated in acne lesions. PLoS One 9: 105238

40. Melnik BC (2015) Linking diet to acne metabolomics, inflammation, and comedogenesis: an update. Clin Cosmet Investig Dermatol 8: 371-388.
41. Kistowska M, Meier B, Proust T, Feldmeyer L, Cozzio A, et al. (2015) Propionibacterium acnes promotes Th17 and Th17/Th1 responses in acne patients. J Invest Dermatol 135: 110-118.

42. Maddur MS, Miossec P, Kaveri SV, Bayry J (2012) Th17 cells: biology, pathogenesis of autoimmune and inflammatory diseases, and therapeutic strategies. Am J Pathol 181: 8-18.

43. Wang P, Thevenot P, Saravia J, Ahlert T, Cormier SA (2011) Radical-containing particles activate dendritic cells and enhance Th17 inflammation in a mouse model of asthma. Am J Respir Cell Mol Biol 45: 977-983.

44. Muralidharan S, Mandrekar P (2013) Cellular stress response and innate im mune signaling: integrating pathways in host defense and inflammation. $J$ Leukoc Biol 94: 1167-1184.

45. Picardo M, Ottaviani M, Camera E, Mastrofrancesco A (2009) Sebaceous gland lipids. Dermatoendocrinol 1: 68-71.

46. Chiba K, Yoshizawa K, Makino I, Kawakami K, Onoue M (2001) Changes in the levels of glutathione after cellular and cutaneous damage induced by squalene monohydroperoxide. J Biochem Mol Toxicol 15: 150-158.

47. Ottaviani M, Alestas T, Flori E, Mastrofrancesco A, Zouboulis CC, et al (2006) Peroxidated squalene induces the production of inflammatory mediators in HaCaT keratinocytes: a possible role in acne vulgaris. J Invest Dermatol 126: 2430-2437.

48. Zouboulis CC (2009) Zileuton, a new efficient and safe systemic anti-acne drug. Dermatoendocrinol 1: 188-192.

49. Grange PA, Chéreau C, Raingeaud J, Nicco C, Weill B, et al. (2009) Production of superoxide anions by keratinocytes initiates $P$ acnes-induced inflammation of the skin. PLoS Pathog 5: 1000527.

50. Sandoval LF, Hartel JK, Feldman SR (2014) Current and future evidence-based acne treatment: a review. Expert Opin Pharmacother 15: 173192

51. Elsaie ML, Abdelhamid MF, Elsaaiee LT, Emam HM (2009) The efficacy of topical $2 \%$ green tea lotion in mild-to-moderate acne vulgaris. J Drugs Dermatol 8: 358-364.

52. Sharquie KE, Noaimi AA, Al-Salih MM (2008) Topical therapy of acne vulgaris using $2 \%$ tea lotion in comparison with $5 \%$ zinc sulphate solution. Saudi Med J 29: $1757-1761$

53. Dreno B, Foulc P, Reynaud A, Moyse D, Habert H, et al. (2005) Effect of zinc gluconate on propionibacterium acnes resistance to erythromycin in patients with inflammatory acne: in vitro and in vivo study. Eur J Dermatol 15: 152-155.

54. Niren NM, Torok HM (2006) The Nicomide Improvement in Clinical Outcomes Study (NICOS): results of an 8-week trial. Cutis 77: 17-28.

55. Kim J, Ko Y, Park YK, Kim NI, Ha WK, et al. (2010) Dietary effect of lactoferrin-enriched fermented milk on skin surface lipid and clinical improvement of acne vulgaris. Nutrition 26: 902-909.

56. Kobayashi M, Yamamoto M (2006) Nrf2-Keap1 regulation of cellular defense mechanisms against electrophiles and reactive oxygen species. Adv Enzyme Regul 46: 113-140

57. Chan K, Han XD, Kan YW (2001) An important function of Nrf2 in combating oxidative stress: detoxification of acetaminophen. Proc Natl Acad Sci USA 98: 4611-4616.

58. Kumar H, Kim IS, More SV, Kim BW, Choi DK (2014) Natural product-derived pharmacological modulators of Nrf2/ARE pathway for chronic diseases. Nat Prod Rep 31: 109-139.

59. Bowe WP, Joshi SS, Shalita AR (2010) Diet and acne. J Am Acad Dermatol 63: 124-141.

60. Cordain $L$ (2005) Implications for the role of diet in acne. Semin Cutan Med Surg 24: 84-91. 